\title{
Influence of higher Fock states in light-cone gauge theories
}

\author{
S. Dalley \\ Theory Division, CERN, CH-1211 Geneva 23, Switzerland \\ (Received 20 April 1998; published 16 September 1998)
}

\begin{abstract}
In the light-cone Fock state expansion of gauge theories, the influence of non-valence states may be significant in precision non-perturbative calculations. In two-dimensional gauge theories, it is shown how these states modify the behavior of the light-cone wave function in significant ways relative to endemic choices of variational ansatz. Similar effects in four-dimensional gauge theories are briefly discussed.
\end{abstract}

[S0556-2821(98)07520-1]

PACS number(s): 11.15.Tk

\section{INTRODUCTION}

Light-cone quantization is believed to be an especially efficient Hamiltonian method for dealing with many-body relativistic field theory [1]. One of the tenets of this method is that the valence approximation to a system of light-cone partons provides a much better approximation than the analogous Tamm-Dancoff truncation in other quantization schemes. This chiefly follows from the peculiar inverse relation between light-cone momentum and free energy:

$$
k^{-}=\frac{m^{2}+\mathbf{k}^{2}}{2 k^{+}} .
$$

Here, $k^{ \pm}=\left(k^{0} \pm k^{3}\right) / \sqrt{2}$ are the light-cone momentum and energy, $\mathbf{k}=\left(k^{1}, k^{2}\right)$ the transverse momenta, and $m$ the mass of a parton. Since $k^{+}>0$ and is conserved, to create more partons costs significant energy because the new particles carry smaller $k^{+}$than their parent(s). Moreover, one might naively expect wave functions to vanish at small $k^{+}$to ensure finite energy. In this Brief Report it will be shown how non-valence states nevertheless modify the behavior of lightcone wave functions, including the valence sector, when one or more parton momenta are small. Rather than wave functions simply vanishing at the boundaries of phase space, the requirements of finite energy in general lead to cancellations between the valence and non-valence (sea) components of bound states. This phenomena was recently analyzed for QCD in Ref. [2], where it was shown to be responsible, at least perturbatively, for the rising Regge behavior of hadronic structure functions at small momentum fraction $x$. In this work some implications for non-perturbative calculations will be demonstrated. The relevant effects may prove significant in precision calculations with variational bases. The basic physical ideas can be illustrated by twodimensional gauge theories. Implications for fourdimensional gauge theories will be discussed later.

\section{TWO-DIMENSIONAL GAUGE THEORIES}

The results to be discussed in this section will in fact be true for any two-dimensional gauge theory exhibiting pair production. Rather than attempting to cover all particular cases at once, we proceed by using familiar examples. The generalization will easily be seen to follow from the simple structure of two-dimensional gauge theories. Therefore, consider the standard two-dimensional gauge theory with fermion fields $\Psi$ and action

$$
S=\int d x^{0} d x^{1} \mathrm{i} \bar{\Psi} \gamma_{\alpha} D^{\alpha} \Psi-m \bar{\Psi} \Psi-\frac{1}{4 e^{2}} F_{\alpha \beta} F^{\alpha \beta},
$$

where representation and color indices are suppressed for the moment, for generality. Using a chiral representation of the two-dimensional $\gamma$ matrices in the light-cone gauge $A_{-}$ $=\left(A_{0}-A_{1}\right) / \sqrt{2}=0$, we are left with $A_{+}=\left(A_{0}+A_{1}\right) / \sqrt{2}$ and the left-moving $\psi_{L}$ and right-moving $\psi_{R}$ components of $\Psi$. $A_{+}$and $\psi_{L}$ are found to be constrained variables with respect to light-cone time $x^{+}=\left(x^{0}+x^{1}\right) / \sqrt{2}$, and can be eliminated by their equations of motion to yield a light-cone Hamiltonian

$$
P^{-}=\int d x^{-} \frac{m^{2}}{2} \psi_{R}^{*} \frac{1}{\mathrm{i} \partial_{-}} \psi_{R}+\frac{e^{2}}{2} J^{+} \frac{1}{\left(\mathrm{i} \partial_{-}\right)^{2}} J^{+} .
$$

Here $x^{-}=\left(x^{0}-x^{1}\right) / \sqrt{2}$ and $J^{+}$is the right-moving current $J^{+}=\psi_{R} \psi_{R}^{*}$. (The same form of Hamiltonian results for bosons $\phi$, with an appropriate current $J^{+}=\mathrm{i} \phi^{*} \stackrel{\vec{\partial}}{-}_{-} \phi$.) These theories generically exhibit linear confinement, since the Coulomb potential is linear in one space dimension. The Hamiltonian (2.2) can be diagonalized in the light-cone Fock space at fixed $x^{+}$, constructed from the Fourier modes of $\psi_{R}$ :

$$
\psi_{R}\left(x^{-}\right)=\frac{1}{\sqrt{2 \pi}} \int_{0}^{\infty} d k\left[b(k) \mathrm{e}^{-\mathrm{i} k x^{-}}+d^{\dagger}(k) \mathrm{e}^{\mathrm{i} k x^{-}}\right] .
$$

A (non-baryonic) eigenfunction of $P^{-}$with total momentum $P^{+}$is of the form 


$$
\begin{aligned}
\left|\Psi\left(P^{+}\right)\right\rangle= & \int_{0}^{P^{+}} d k_{1} d k_{2} \delta\left(P^{+}-k_{1}-k_{2}\right) \\
& \times f_{2}\left(k_{1}, k_{2}\right) b^{\dagger}\left(k_{1}\right) d^{\dagger}\left(k_{2}\right)|0\rangle \\
& +\frac{1}{2} \int_{0}^{P^{+}} d k_{1} d k_{2} d k_{3} d k_{4} \delta\left(P^{+}-\sum_{i=1}^{4} k_{i}\right) \\
& \times f_{4}\left(k_{1}, k_{2}, k_{3}, k_{4}\right) b^{\dagger}\left(k_{1}\right) b^{\dagger}\left(k_{2}\right) d^{\dagger}\left(k_{3}\right) d^{\dagger}\left(k_{4}\right)|0\rangle \\
& +\cdots .
\end{aligned}
$$

Ellipses indicate higher Fock states $f_{n}, n>4$.

Projecting $2 P^{+} P^{-}\left|\Psi\left(P^{+}\right)\right\rangle=M^{2}\left|\Psi\left(P^{+}\right)\right\rangle$onto specific Fock states, where the eigenvalue $M^{2}$ is the invariant mass squared of the system, one derives coupled integral equations for the wave function components $f_{n}$. Let us consider the familiar example of two-dimensional QED (massive Schwinger model at $\theta=0),{ }^{1}$ which in the approximation that retains only $f_{2}$ and $f_{4}$ reads $[3,4]$

$$
\begin{aligned}
M_{2} f_{2}(x, 1-x)= & \frac{m^{2}}{x(1-x)} f_{2}(x, 1-x) \\
& +\frac{e^{2}}{\pi} \int_{0}^{1} d y \frac{f_{2}(x, 1-x)-f_{2}(y, 1-y)}{(x-y)^{2}} \\
& +\frac{e^{2}}{\pi} \int_{0}^{1} d f_{2}(y, 1-y) \\
& +\frac{e^{2}}{\pi} \int_{0}^{1} d y_{1} d y_{2} d y_{3} \delta\left(y_{1}+y_{2}+y_{3}-x\right) \\
& +\frac{f_{4}\left(y_{1}, y_{2}, y_{3}, 1-x\right)}{\left(x-y_{1}\right)^{2}} \\
& -\frac{e^{2}}{\pi} \int_{0}^{1} d y_{2} d y_{3} d y_{4} \delta\left[y_{2}+y_{3}+y_{4}\right. \\
& -x)] \frac{f_{4}\left(x, y_{2}, y_{3}, y_{4}\right)}{\left(1-x-y_{4}\right)^{2}} \\
& \\
& \\
& \\
&
\end{aligned}
$$

\footnotetext{
${ }^{1}$ Some observations related to those made in this paper have recently been given in connection with the $\theta$-dependence of the massive Schwinger model [5].
}

$$
\begin{aligned}
M^{2} f_{4}\left(x_{1}, x_{2}, x_{3}, x_{4}\right) \\
=m^{2} \sum_{i=1}^{4} \frac{1}{x_{i}} f_{4}\left(x_{1}, x_{2}, x_{3}, x_{4}\right)+\frac{e^{2}}{2 \pi} A\left(x_{1}, x_{2}, x_{3}\right) \\
\quad+\frac{e^{2}}{\pi} \int_{0}^{1} d y_{1} d y_{2}\left\{\delta\left[y_{1}+y_{2}-\left(x_{1}+x_{2}\right)\right]\right. \\
\left.\quad \times \frac{f_{4}\left(y_{1}, y_{2}, x_{3}, x_{4}\right)-f_{4}\left(x_{1}, x_{2}, x_{3}, x_{4}\right)}{\left(x_{1}-y_{1}\right)^{2}}+\text { similar }\right\},
\end{aligned}
$$

where and $x_{i}=k_{i} / P^{+}, x_{4}=1-x_{1}-x_{2}-x_{3}$ and

$$
\begin{aligned}
A\left(x_{1}, x_{2}, x_{3}\right) & \\
= & \frac{f_{2}\left(1-x_{3}, x_{3}\right)-f_{2}\left(x_{2}, 1-x_{2}\right)}{\left(1-x_{2}-x_{3}\right)^{2}} \\
& +\frac{f_{2}\left[x_{1}+x_{2}+x_{3}, 1-\left(x_{1}+x_{2}+x_{3}\right)\right]-f_{2}\left(x_{1}, 1-x_{1}\right)}{\left(x_{2}+x_{3}\right)^{2}} \\
& +\frac{f_{2}\left(x_{2}, 1-x_{2}\right)-f_{2}\left[x_{1}+x_{2}+x_{3}, 1-\left(x_{1}+x_{2}+x_{3}\right)\right]}{\left(x_{1}+x_{3}\right)^{2}} \\
& +\frac{f_{2}\left(x_{1}, 1-x_{1}\right)-f_{2}\left(1-x_{3}, x_{3}\right)}{\left(1-x_{1}-x_{3}\right)^{2}} .
\end{aligned}
$$

The many "similar', contributions to the last term in (2.10) are enumerated in Ref. [4], but are unimportant for our purposes. At this point it is important to stress the distinction between the component $f_{2}$ to the solution of such equations in the valence approximation, which sets $f_{n}=0$ for $n>2$, and the component $f_{2}$ to the solution when some or all of the higher Fock states $f_{n}$ are retained. As is shown below, these have different high-energy behavior in general.

Like any wave function, the components $f_{n}$ are subject to high energy boundary conditions. From Eq. (1.1), this is a small light-cone momentum boundary. A typical variational ansatz used for the solution of the light-cone bound state integral equations is

$$
f_{n}\left(x_{1}, \ldots, x_{n}\right) \sim x_{1}^{\beta} x_{2}^{\beta} \cdots x_{n}^{\beta} \cdot P\left(x_{1}, \cdots, x_{n}\right)
$$

where $x_{n}=\left(1-\sum_{i=1}^{n-1} x_{i}\right)$ and $P$ is polynomial. The safest procedure is to leave $\beta>0$ as a variational parameter. However, often the following formula is used:

$$
m^{2}=e^{2}(1-\pi \beta \cot \pi \beta) .
$$

This choice is motivated by a study of the high-energy "end point" behavior of $f_{n}\left(x_{1}, \ldots, x_{n}\right)$ as one of the arguments vanishes, $x_{i} \rightarrow 0$. Ignoring terms resulting from a change in the number of partons [pair production (2.8), (2.9)], one deduces that for finite $M^{2}$, as $x \rightarrow 0$ the divergent behavior of the mass (2.5) and Coulomb terms (2.6) must cancel:

$$
\begin{aligned}
& \lim _{x \rightarrow 0}\left(\frac{m^{2}}{x} f_{2}(x, 1-x)+\frac{e^{2}}{\pi} \int_{0}^{1} d y \frac{f_{2}(x, 1-x)-f_{2}(y, 1-y)}{(x-y)^{2}}\right) \\
& \quad=\text { finite. }
\end{aligned}
$$


(The Coulomb term is dominated by the region of asymptotically small momentum transfer $y \sim x$.) This depends only upon the end point behavior of $f_{2}$ and leads to Eq. (2.13), as was first shown by 't Hooft in large- $N_{c}$ two-dimensional QCD [6] (for which only $f_{2}$ exists). The same end point behavior can be deduced under similar assumptions for any $f_{n}$ when one of its arguments vanishes. The usual justification given for ignoring pair production (see, e.g., [8]) is based on the assumption that $f_{n}$ does not diverge if three or more momenta vanish. In this case, it is easy to see from Eqs. (2.8), (2.9) by power counting that pair production would be subleading as $x \rightarrow 0$.

In fact, the ansatz (2.12) with (2.13) fails in general to account correctly for the behavior of $f_{n}$ when one momentum vanishes, except for $f_{2}$ in the valence approximation (or more generally, $f_{n}$ in the approximation that sets $f_{m}=0$ for $m>n)$. Furthermore, Eq. (2.12) fails to account for the correct behavior as two or more momenta vanish in $f_{n}$. This follows by examining the behavior of Eqs. (2.5)-(2.10) when two or more momenta vanish. To illustrate these points, let us perform a one-loop calculation in $\mathrm{e} / \mathrm{m}$. In this case, it is easy to see that to leading order in $\mathrm{e} / \mathrm{m}$, the solution to the four-parton equation (2.10) is

$$
f_{4}\left(x_{1}, x_{2}, x_{3}, x_{4}\right)=\frac{e^{2}}{2 \pi} \frac{A\left(x_{1}, x_{2}, x_{3}\right)}{M^{2}-m^{2} \sum_{i=1}^{4}\left(1 / x_{i}\right)}
$$

[note that $\left.M^{2} \sim O\left(m^{2}\right)\right]$. This shows that as two momenta vanish, say $x_{1}$ and $x_{3}$, such that $\lim _{z \rightarrow 0} x_{1}, x_{3} \propto z$, then $f_{4}$ is non-zero and finite, which is incompatible with Eq. (2.12). Furthermore, as three momenta vanish, say $\lim _{z \rightarrow 0} x_{1}, x_{2}, x_{3}$ $\propto z$, then $f_{4}$ diverges as $z^{\beta-1}$ if $f_{2}(z, 1-z) \sim z^{\beta}$, which is also incompatible with Eq. (2.12). Therefore, rather than vanishing at the corners of phase space, we find relations between wave function components in each Fock sector, showing in particular that the valence and the sea are not analytically independent. Substituting $f_{4}(2.15)$ into the twoparton equation (2.5)-(2.9), one obtains an effective valence equation. In the limit of small $x$ the singular part of this equation reads

$$
\begin{aligned}
\lim _{x \rightarrow 0}\left\{\frac{e^{2}}{\pi} \int_{0}^{1} d y \frac{f_{2}(x, 1-x)-f_{2}(y, 1-y)}{(x-y)^{2}}\left(1-\frac{e^{2}}{2 m^{2} \pi} I(x / y)\right)\right. \\
\left.+\frac{m^{2}}{x} f_{2}(x, 1-x)\right\}=\text { finite } \\
I(w)=\int_{0}^{1} d v\left\{\frac{1}{[1 / v(1-v)]+w-1}\left(1-\frac{(1-w)^{2}}{[1-v(1-w)]^{2}}\right)\right. \\
\left.+\quad \frac{1}{(1+w / v(1-w))^{2}(w+1 / 1-v)}\right\} .
\end{aligned}
$$

Comparing with Eq. (2.14), this demonstrates that the neglect of pair production is unjustified in the determination of $\beta$, and that Eq. (2.13) will not be correct in general.

To summarize, the ansatz (2.12) with (2.13) correctly accounts for the behavior of $f_{n}\left(x_{1}, \ldots, x_{n}\right)$ as one $x_{i} \rightarrow 0$ only in the approximation which sets $f_{m}=0$ for $m>n$. More generally $\beta$ will be renormalized from the value given by Eq. (2.13). Equation (2.12) fails to account for the behavior as two or more momenta vanish.

First order $\mathrm{e} / \mathrm{m}$ perturbation theory and a 4-particle Tamm-Dancoff Fock-space truncation were used above, but it is easy to check that the phenomenon is not special to these choices. It follows by power counting in the bound state integral equations when various momenta $x_{i}$ are made to vanish, i.e., one does not need to solve the equations to reach the conclusions. Moreover, it is also easy to check that since it only depends upon the current-current structure of interactions given in Eq. (2.2), it will occur in any two-dimensional gauge theory with pair production; in particular, for fundamental representations at finite $N_{c}[10]$ and adjoint representations [11]. In the massive Schwinger model, typically a one percent error in $\beta$ can lead to a similar size error in $M^{2}$ estimated from variational ansatz, which is significant at the level of precision of current computations [4,12]. The error is small in this case because the valence approximation becomes exact in the large and small $m$ limits. In cases where the latter is not true, such as boson or Majorana fermion representations, the effects maybe somewhat larger, especially for small $m$.

The algebraic manipulations have a simple underlying physical explanation. Higher Fock states renormalize the Coulomb coupling at asymptotically small momentum transfer through vacuum polarization and vertex renormalization. In general, the parton mass $m^{2}$ may also be renormalized. Sector-dependent renormalizations similar to these are wellknown in the Tamm-Dancoff treatment of light-cone boundstate integral equations [9]. However, it does not seem to have been generally recognized that in gauge theories, higher Fock components of the wave function may diverge at the corners of phase space. Via loop integrations which are naively of measure zero [viz. Eq. (2.8) as $x \rightarrow 0$ ], renormalization results, e.g., in formulas such as Eq. (2.13).

A significant effect of these renormalizations might be expected in theories which exhibit complete screening in some limit. This is the case for $m \rightarrow 0$ in two-dimensional QED, for example. In this limit the string tension is known to vanish as $\sigma \sim e m[7] .^{2}$ Pair production effects now completely screen the linear potential as $m \rightarrow 0$, resulting in the Higgs mechanism and the production of Schwinger's massive photon in the physical spectrum. If we assume a similar renormalization, in the bound state integral equations, of the Coulomb coupling at asymptotically small momentum transfer $e^{2} \rightarrow e_{R}^{2} \sim e m$ (this may not be entirely justified since the sources are no longer heavy), one naively finds from Eq. (2.13) that $\beta \sim \sqrt{m}$ as $m \rightarrow 0$, rather than $\beta \sim m$. In any case, since it is $e_{R}$ which is relevant to the determination of $\beta$, rather than $e$, this emphasizes the need to properly account

\footnotetext{
${ }^{2}$ This result was established by bosonization. In principle there can be a renormalization of the parameter $m / e$ in the bosonization approach relative to a fermionic basis, which prevents direct comparison with light-cone results in such a basis.
} 
for renormalization effects due to higher Fock states, before attempting to accurately solve light-cone gauge theories by variational ansatz. It may have some bearing on the small discrepancies in results found between bosonization and light-cone quantization of two-dimensional QED [12]. More generally, long-distance quantities, like gauge theory string tension measurements, will be especially affected by singular behavior of higher Fock states.

\section{FOUR-DIMENSIONAL GAUGE THEORIES}

The considerations above are relevant in QCD to the behavior of partons carrying small light-cone momentum fraction $x$ of the hadron. The transverse lattice color-dielectric formulation of pure QCD is conceptually similar to twodimensional gauge theories with adjoint boson representations [13]. This is especially true in the large- $N_{c}$ limit, when an exact dimensional reduction to two-dimensional gauge theory takes place [14]. Use of variational wave functions in some form seems unavoidable to obtain satisfactory numerical convergence with this method. The job of implementing the subtleties described in the previous section for higher Fock states looks quite formidable. However, a hybrid method introduced in Ref. [14], which combines a variational approach with discretized light-cone quantization (DLCQ) [15], offers a compromise. In this method, which may be applied to any light-cone theory, the parton momentum fractions $x_{i}=t_{i} / K$ are artificially discretized into integers $t_{i}$, such that $\sum_{i=1}^{n} t_{i}=K$. When computing matrix elements of $P^{-}$in Fock space, spectator partons retain their discrete momentum $t_{i}$, while partons involved in an elementary interaction have their momenta projected onto a continuous-momentum wave function basis (see Appendix C of Ref. [14] for details), for evaluation by variational ansatz. For a theory with elementary interactions of order $\leqslant q$ in the number of fields, no more than $q$ partons are involved in such interactions in initial plus final states. This means that that an appropriate variational basis need only be found for a few partons, however many higher Fock states are included in the calculations. Other variational approaches to light- cone QCD break gauge invariance explicitly by giving large constituent masses to quarks and gluons [16]. It is hoped that higher Fock states are strongly suppressed as a result and that perturbative renormalization theory can be performed.

Finally, it should be emphasized that the singular behavior of light-cone wave functions, when two or more lightcone momentum fractions $x_{i}$ vanish, has observable implications for the small Bjorken- $x$ region of hadronic structure functions. This behavior is determined from the high-energy boundary condition on light-cone wave functions in QCD [2], which can be easily solved perturbatively and again relates Fock space sectors with differing numbers of partons. The definition of the distribution function for a parton of momentum fraction $x_{1}$ is

$$
\begin{aligned}
Q\left(x_{1}\right)= & \sum_{n} \int[d \mathbf{k}] \int d x_{2} \cdots d x_{n} \delta\left(1-\sum_{i=1}^{n} x_{i}\right) \\
& \times\left|f_{n}\left(x_{1}, x_{2}, \ldots, x_{n}\right)\right|^{2} .
\end{aligned}
$$

$[d \mathbf{k}]$ indicates the integration over all parton transverse momenta upon which $f_{n}$ also depends in four dimensions (this is absent in two dimensions). Generalizing the analysis of the previous section to all $n$, one finds that the wave functions of two-dimensional gauge theories diverge slower than $x^{-(s-2)}$ when $s \geqslant 3$ light-cone momenta are of order $x \rightarrow 0$, and are otherwise finite. By power counting, this means that they are not singular enough to prevent $Q(x \rightarrow 0)$ from vanishing in two dimensions. In four-dimensional gauge theories however, it is possible, via the quark-gluon-anti-quark vertex for example, for $f_{n}$ to diverge already when only two parton momenta are vanishing, leading to $\log x$ contributions to $Q(x \rightarrow 0)[2,17]$. The accumulation of such logarithms is thought to lead to the power law rise (Regge behavior) exhibited by hadronic structure functions at small $x$.

\section{ACKNOWLEDGMENTS}

I would like to thank B. van de Sande for discussions. This work is supported by CERN.
[1] S. J. Brodsky, H-C. Pauli, and S. Pinsky, Phys. Rep. 301, 299 (1998).

[2] F. Antonuccio, S. J. Brodsky, and S. Dalley, Phys. Lett. B 412, 104 (1997).

[3] H. Bergknoff, Nucl. Phys. B122, 215 (1977).

[4] Y. Mo and R. J. Perry, J. Comput. Phys. 108, 159 (1993).

[5] M. Burkardt and K. Harada, Phys. Rev. D 57, 5950 (1998).

[6] G. 't Hooft, Nucl. Phys. B75, 461 (1974).

[7] S. Coleman, R. Jackiw, and L. Susskind, Ann. Phys. (N.Y.) 93, 267 (1975)

[8] K. Hornbostel, Ph.D. thesis, Stanford, 1988.

[9] R. J. Perry and A. Harindranath, Phys. Rev. D 43, 4051 (1991)
[10] K. Hornbostel, S. J. Brodsky, and H-C. Pauli, Phys. Rev. D 41, 3814 (1990).

[11] S. Dalley and I. R. Klebanov, Phys. Rev. D 47, 2517 (1993).

[12] K. Harada, T. Heinzl, and C. Stern, Phys. Rev. D 57, 2460 (1998), and references therein.

[13] W. A. Bardeen and R. B. Pearson, Phys. Rev. D 14, 547 (1976).

[14] S. Dalley and B. van de Sande, Phys. Rev. D 56, 7917 (1997).

[15] H. C. Pauli and S. J. Brodsky, Phys. Rev. D 32, 1993 (1985); 32, 2001 (1985).

[16] K. G. Wilson et al., Phys. Rev. D 49, 6720 (1994).

[17] A. H. Mueller, Nucl. Phys. B415, 373 (1994). 